\title{
A Modified Fault Detection Bayesian Learning Model For Inter Connected Vehicle Networks

\author{
Syed Umar ${ }^{1}$, Tadele Debisa Deressa ${ }^{2}$, Bodena Terfa ${ }^{3}$
} \\ ${ }^{1}$ Professor, Department of Computer Science, Wollega University, Nekemte, Ethiopia \\ ${ }^{2}$ Lecture, Department of Computer Science, Wollega University, Nekemte, Ethiopia \\ ${ }^{3}$ Lecturer \& HOD, Department of Informatics, Wollega University, Nekemte, Ethiopia
}

\section{Article Info}

Volume 8 Issue 2

Page Number: 182-189

Publication Issue :

March-April-2021

\section{Article History}

Accepted : 25 March 2021

Published : 31 March 2021

\section{ABSTRACT}

Currently an important worldwide web, The IoT represents the biggest connected vehicle network of all, but will evolve into a much larger network of connected vehicles. Though a concept promising, the combination of different enabling frequencies does pose various intrinsic and defining challenges in the form of communication systems like privacy and protection. It is also important to establish an effective and dependable strategy to access information for solutions which emerge from increasingly complex vehicle and data systems because of the proliferation of wireless medium. In this article, we provide and improve a new algorithm known as Advanced Fault Detection and Management with Bayesian Network techniques, in which we intend to locate and adjust spatial vehicle faults in real time. Often, we apply measurement method to discover the most effective fault detection methodology, which is the turning point. A sequence of recent studies illustrated findings shows that the suggested approaches include fault detection and correction utilizing tools accessible previously.

Keywords : Battery Storage, Super capacitors, Renewable Resources, Wind Power, Supervisory Controller, Battery Lifetime

\section{INTRODUCTION}

With the rapid implementation of Internet of Things (IoT) over different billion things which are communicated with worldwide by 2020. Based on sensed information taken from specific persons monitor and ambient outside environment. Intelligent vehicles with sensory capabilities and communication with other vehicles perform different advanced functions to implement automatic actions in [1-7]. Because of remarkable implementation of vehicular and wearable technologies, intelligent vehicles are extracted independently. Integrating intelligent vehicles interactively promising different urban related applications environments. To improve advance driver assistance systems field in automobile technology, which can be take the advantage of potential wearable intelligent systems developed by several automobile manufacture applications.

However increasing the integrating intelligent vehicle system faces different variety of unproductive 
challenges and emerging technologies developed by internet of things applications developed by wide area networks. Because of intrinsic characteristics in wide range of networks, IoT can't apply directly on vehicle communication systems. Communication systems describe heterogeneous traffic patterns with different Quality of service (QoS) parameters long with different wearable and vehicle devices generated from different Wide range long networks. Specifically, mobility of different human beings describe dynamic structure of network which describes the flexibility and reliability. Because of wireless network communication systems, various vehicle and wearable technologies increased respectfully.

Based on existing literature, its mainly focus on application development of vehicle and wearable vehicle technologies. There are different types approaches/achievements are applied to identify faults of different connected vehicles present in integrated vehicle system described in [6][7]. Current research behind this concept, different researchers mainly focus and diagnoses abnormal vehicle which consider communication aspect with respect to loss of communication. To this end, we implement and use a Bayesian Network Architecture, which will be useful for real-time spatial vehicle fault detection and rectification. Often, we apply measurement method to discover the most effective fault detection methodology, which is the turning point. Using this fault detection and modification system for different communicated vehicles with high accuracy in classification of fault related vehicles based on previous communication of each vehicle present in wearable technologies. Main contribution of proposed approach as follows:

In this proposed approach, implement Bayesian Network model to explore vehicle information based on index of each vehicles, implement threshold based fault detection procedure for estimate the true or false positions of vehicles. To perform these representations, in this approach, first implement preprocessing step (training application with different vehicles with their positions) to improve quality of service parameter sequences present in Bayesian network model. Compare test results of proposed approach with traditionally approaches with respect to minimize faults and attack sequences in distributed vehicular ad hoc networks.

\section{Different Promising Characteristics of WeVe}

Figure 1 portrays the situation of $\mathrm{WeVe}$ in urban conditions. Brilliant wearables (e.g., smart glasses, ECG, savvy, movement sensor) regularly screen the physiological data of the client (either drivers or on the other hand travelers in vehicles or walkers on the lanes) to give medicinal services administrations discussed in [8-13]. Other than the wearable sensors, savvy vehicles are prepared with a large number of car installed sensors (e.g., front light range sensor, reflect sensor, transmission sensor, fuel level sensor), which are interconnected to screen the status of the vehicle.

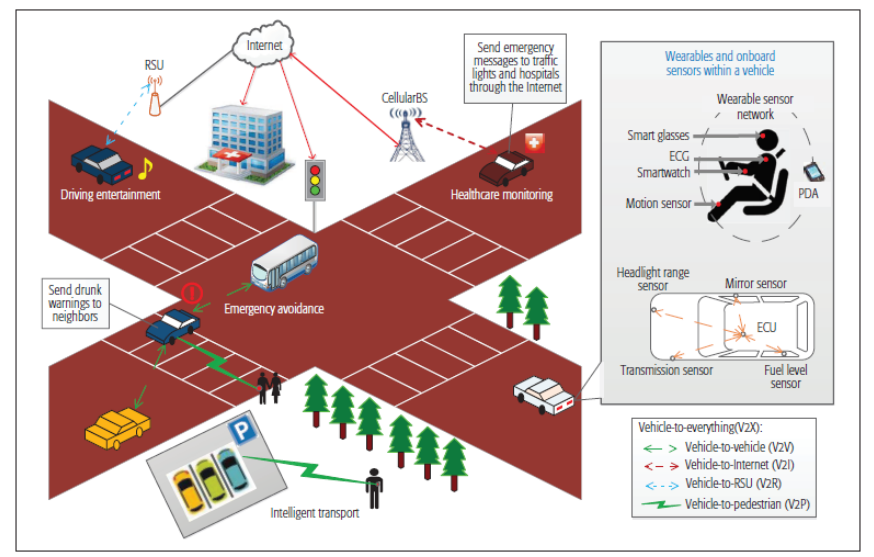

Figure 1. Description of WeVe urban environment for different vehicle communications.

On the road, vehicles are associated with different vehicles, the Internet, roadside units (RSUs), and people on foot. WeVe empowers vehicular advances to be more mindful of drivers and travellers. By methodically joining the correspondence advances created for savvy wearable and keen vehicles, Wave is imagined to have the option to naturally incorporate 
what's more, deal with the exhaustive information going from vehicle data, traffic data, and ecological data, to driver and traveller data, in order to give more accommodation and further encourage our day by day life.

\section{Basic Problem Formation}

In this section, we describe the basic formation of problem with respect to fault identification and modification procedure for communicated vehicles to formalize description Bayesian network model to perform learning process for different vehicle information.

\subsection{Formation of Problem}

Assume that $\mathrm{N}$ sensors are utilized to screen $\mathrm{N}$ files of a vehicle and report the perception esteems once per schedule opening. We use $\mathrm{fk}, \mathrm{t}$ to indicate the perception of kth sensor and gk,t to mean the real estimation of the kth observed record at time $t$. The issue recognition issue is to identify all sensor defective perceptions that are unique in relation to the real estimations of the observed lists, and the fixing issue is to revamp the defective tactile readings of checked records by the evaluated qualities dependent on comparing sensor readings in [14][15]. To assess the presentation of the proposed issue recognition and fixing plan, we characterize four measurements:

1) deficiency location rate $\alpha$ : it is the extent of shortcomings that are effectively recognized.

2) false caution rate $\beta$ : it is the extent of right information that are erroneously analyzed as shortcomings.

3) right deficiency fixing rate $\gamma$ : it is the extent of issues that are effectively fixed.

4) back deficiency rate $\sigma$ : it is the extent of issues (counting the two blames that are not effectively fixed and right information that are inaccurately analyzed and fixed as issues) after the shortcoming location and fixing process.

\subsection{Fault identification Procedure based on Bayesian Network Model}

The dynamic Bayesian system [1] is a successive augmentation of Bayesian system, which joins the fleeting connection between nearby time cuts and the causal relationship in a similar time cut, at that point makes dynamic investigation by quantitative thinking described in [16][17]. The DBN model essentially contains shrouded state factors that speak to reality conditions of the framework and watched state factors that speak to the estimations of reality states with the supposition that the DBN fulfils two conditions: (1) the topology structure of the DBN does not change after some time; (2) the DBN fulfils the primary request Markov condition. The DBN can't be viewed as an all-encompassing model of Bayesian system in time arrangement if the these two presumptions are not fulfilled.

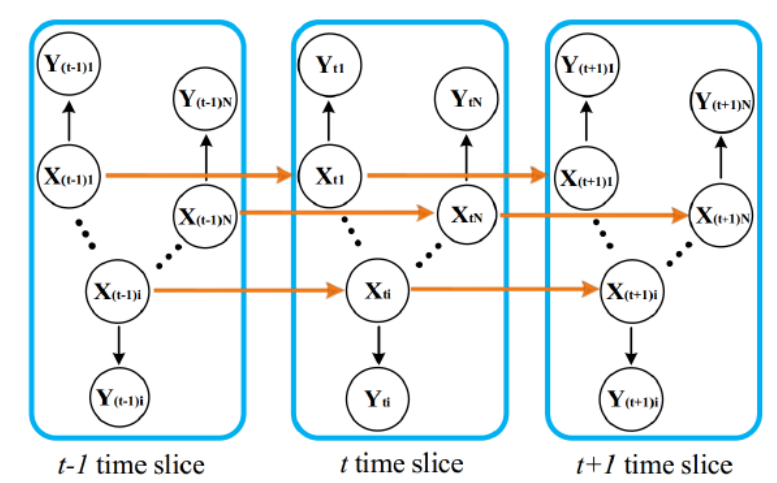

Figure 2. Basic representation of Bayesian network model with communicated vehicles.

The DBN can be characterized as a tuple $(\mathrm{B} 0, \mathrm{~B} \rightarrow)$, where $\mathrm{B} 0$ speaks to the underlying system structure of the model, $\mathrm{B} \rightarrow$ is the DBN move system structure in [17]. We formalize a dynamic Bayesian system model appeared in Fig. 2 to depict the checking procedure of associated vehicles during $\mathrm{T}$ time cuts, where $\mathrm{Xt} 1, \mathrm{Xt} 2, \cdot, \mathrm{XtN}(1 \leq \mathrm{t} \leq \mathrm{T})$ speak to the real estimations of $\mathrm{N}$ observed records at time $\mathrm{t}$, and $\mathrm{Yt} 1, \mathrm{Yt} 2, \cdot \mathrm{YtN}$ speak to the relating tangible readings. We use $\mathrm{Xt}$ to mean the vector $(\mathrm{Xt1}, \cdot \mathrm{XtN})$ and $\mathrm{Yt}$ to signify $(\mathrm{Yt} 1, \cdot \mathrm{YtN})$. 


\section{Fault Identification and Modification for Connected Vehicles}

This section describes the basic representation of vehicle communication, Figure 3 describes representation implementation procedure with respect to different types vehicle communication to monitoring vehicle speed and location based on index values.

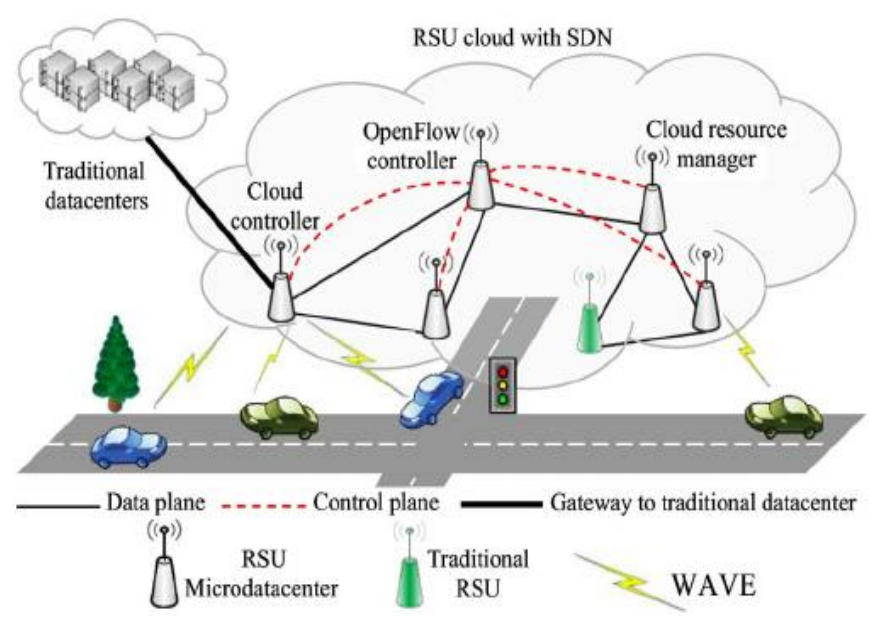

Figure 3. Basic representation of controlling and detection of different vehicles in distributed ad hoc networks.

Fig. 3 demonstrates the system engineering of our observing for vehicle's files like speed and area and it also represents architecture with modules. The continuous traffic information are transmitted by interchanges between vehicle to Internet, vehicle to vehicle, vehicle to individual and vehicle to street foundation to understand the successful use of vehicle characteristic data, static and dynamic data on the system stage, adequately screen vehicle running status and give far reaching administration to vehicles in [12][15]. Vehicles can't just gather information of their own driving status through GPS, RFID and savvy detecting gadgets, yet additionally gather pertinent information from neighbouring vehicles. The unwavering quality of vehicle information is significant in light of the fact that inconsistent data may cause genuine outcomes. So deficiency recognition and fixing are fundamental before these information are utilized for data catch.

\subsection{Learning of parameter and structure based on Bayesian Network Model}

For efficient fault identification and modification using the Bayesian network model, pre-condition and architecture implementation of dynamic Bayesian network (DBN) [9-10] which is performed based on trained data available in different simulated vehicle information. Basically it is not possible to explore actual vehicle information with monitoring indexes; we explore the data generated from sensor reading present in trained data.

\subsubsection{Procedure Used for DBN}

Basic structure procedure is obtain from Bayesian network structure $G$ which consists trained data $Z$, in this Particle swarm optimization (PSO) for proposed Bayesian network structure representation shown in algorithm 1.

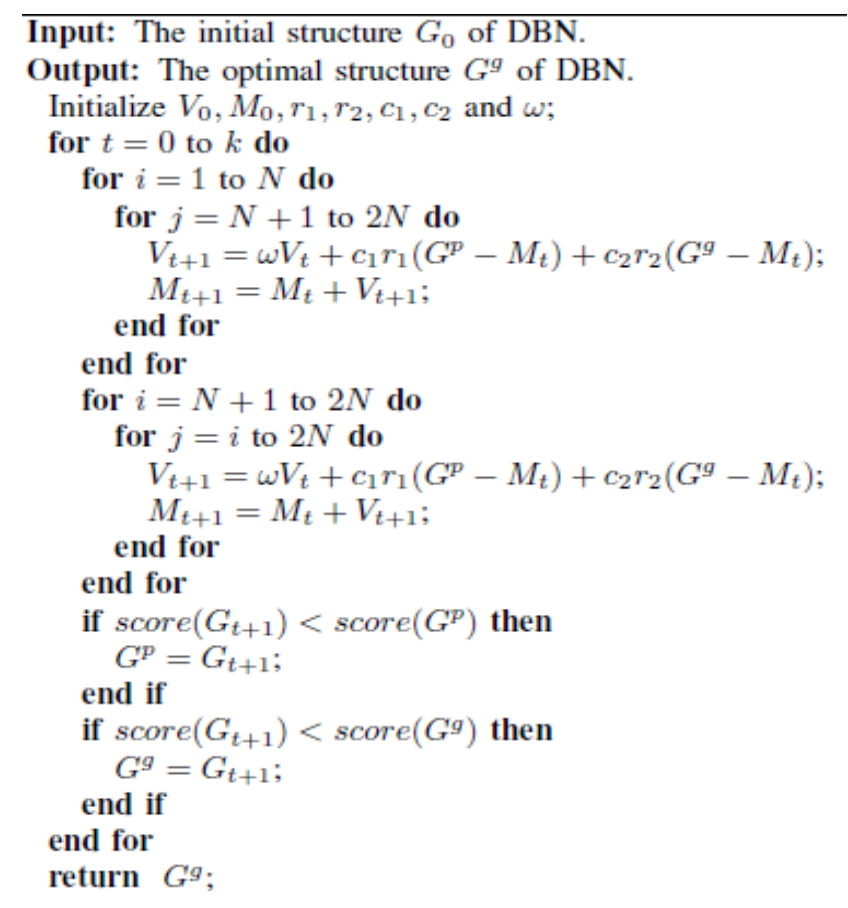

Algorithm 1 Particle swarm optimization procedure to handle structure learning of network 
To develop this implementation for accessing information from different vehicles with two following matrixes i.e. adjacency matrix, and position matrix of Bayesian network structure. Describe the velocity matrix $\mathrm{V}$ calculated as $v_{i j}=1$, if directed connection from edges I to $\mathrm{j}$ and added to Bayesian network model. Based on PSO calculation applied on adges velocity matrix to be calculated based on following equations.

$$
V_{k+1}=w V_{k}+c_{1} r_{1}\left(G^{p}-M_{k}\right)+c_{2} r_{2}\left(G^{g}-M_{k}\right)
$$

where $\mathrm{Gp}$ is the nearby ideal structure, $\mathrm{Gg}$ is the worldwide ideal structure, r1 and r2 are arbitrary numbers between 0 also, $1, \mathrm{c} 1$ and $\mathrm{c} 2$ are learning factors, $\omega$ is the inactivity weight used to control the speed of the past cycle sway on the present speed. We select the structure Ge'k which has the littlest score, at that point update $\mathrm{Gp}$ by $\mathrm{Ge}$ ' $\mathrm{k}$ if score(Ge'k ) is not exactly the score of current nearby ideal $\mathrm{Gp}$, and comparatively update $\mathrm{Gg}$ by $\mathrm{Ge}$ 'k if score $\left(\mathrm{Ge}^{\prime} \mathrm{k}\right)$ is not exactly the score of current worldwide ideal Gg. At last, we select the worldwide ideal $\mathrm{Gg}$ as the last learning structure of the dynamic Bayesian system.

\subsubsection{Threshold based Fault identification and Modification}

To decide a sensor perusing Yti is a deficiency or not, we should get the real worth Xti of the ith list at time $\mathrm{t}$ which is unrealistic. On the other hand, we analyze blames by assessing a genuine worth $\mathrm{Hti}$ for Xti utilizing the dynamic Bayesian system model. There are two kinds of flaw identification of vehicles, the first is the constant issue identification, which is to analyze $\mathrm{Yt}$ at the present time $\mathrm{t}$ by the history sensor perusing $\mathrm{Y} 1, \cdot, \mathrm{Yt}-1$, the other is the non ongoing shortcoming discovery, which is to analyze Yt sooner or later $\mathrm{t}$ by the sensor readings $\mathrm{Y} 1, \cdot, \mathrm{Yt}, \cdot, \mathrm{YT}$. For flaw discovery of associated vehicles, we initially compute the likelihood $\mathrm{P}(\mathrm{Xti} \mid \mathrm{Y} 1, \cdot$, YT $)$ for every conceivable estimation of Xti, and afterward select a worth xti which can amplify the likelihood $\mathrm{P}(\mathrm{Xti} \mid \mathrm{Y} 1, \cdot, \mathrm{YT})$ as the assessed worth Hti for Xti . Given an threshold edge is $\delta$, if the deviation of the $\mathrm{Hti}$ and Yti is more prominent than $\delta$, composed as $|\mathrm{Hti}-\mathrm{Yti}|>\delta$, we analyze the sensor perusing Yti as a flaw, and reconstructed Yti as Hti, generally Yti is resolved as a right perception. In the accompanying, we will give detail process for flaw recognition and fixing in light of the figuring's of assessed estimation of Xti which can expand $\mathrm{P}(\mathrm{Xti} \mid \mathrm{Y} 1, \cdot$, YT ).

\section{Experimental Evaluation}

In this section, we describe the experimental results of proposed approach in terms of fault identification and modification with traditional available approaches.

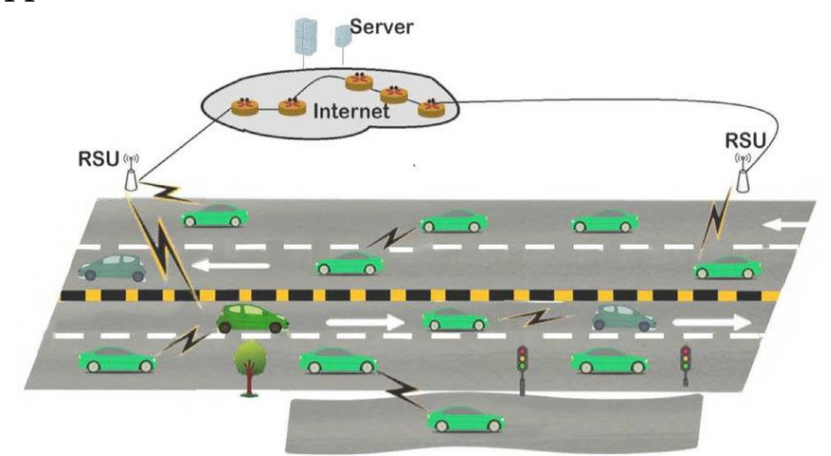

Figure 4. Produced vehicle data from simulated vehicle in wireless vehicular networks

\subsection{Data set Description:}

Simulated Vehicle data taken from different mdf formatted source urls with different scenarios which are developed in wearable vehicle technologies used in wireless network communication. To lead the analyses, we set up a gathering of running information on the turnpike in Xi'an with a Focus vehicle. We begin from the Xidian University, at that point go to the freeway of Xi'an following by the LianHuo freeway, and leave the turnpike from the Huayin tollgate which is acclaimed with the Hua mountain. Our direction is set apart on the guide in Fig. 4. During this procedure, we utilize the ELM327 
demonstrative hardware what's more, the product called Scan Master to get the vehicle information from the OBD interface including speed, rotational speed, temperature, etc. Furthermore, we utilize a synchronous cell phone with GPS to acquire vehicle GPS information. Prior to the examination, we originally set the season of the Scan Master and the cell phone to the intranet system time to guarantee that they can acquire the comparing information simultaneously. Here, we just spotlight on three characteristics: speed, rotational speed, and GPS.

\subsection{Results}

Experimental results of proposed approach with respect to storage of integrated vehicle data with indexes show in figure 5.

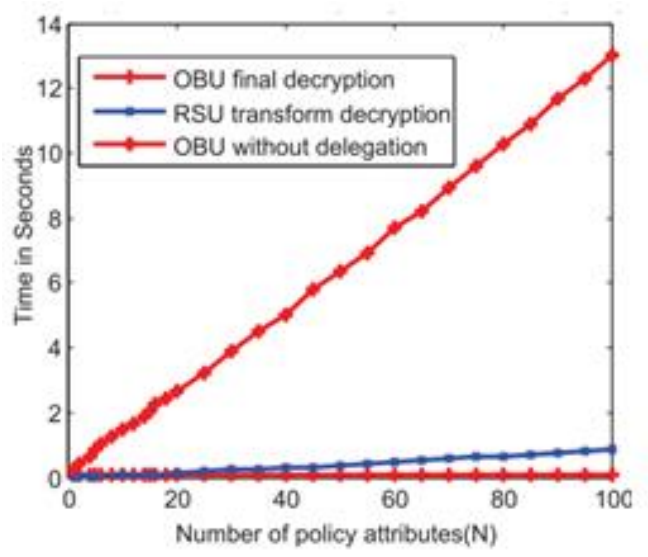

Figure 5. Performance of proposed approach at different index data storages

Figure 5 presents the performance of proposed approach with respect to RSU generated data with delegation and without delegation at different indexes in wireless vehicular ad hoc network communications.

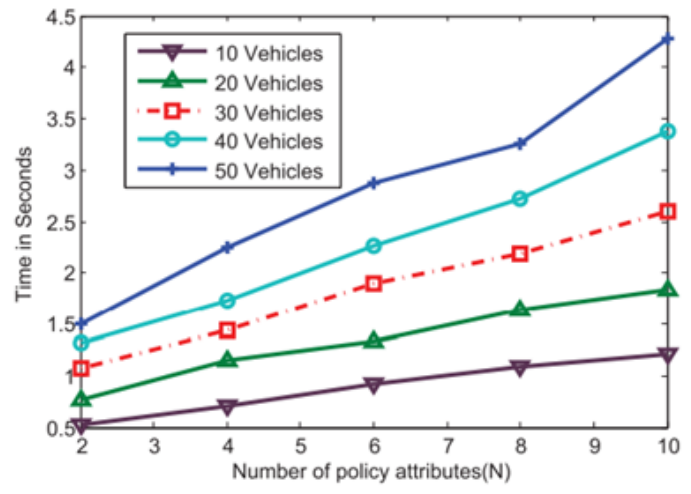

Figure 6. Performance of proposed approach with respect to vehicles processing.
Figure 6 shows the performance of different vehicles at different policy attribute levels with processing of vehicles at different conditions reliable in wireless ad hoc networks.

Fault identification rate with traditional approaches in terms of speed of vehicle data in wireless vehicle communication.

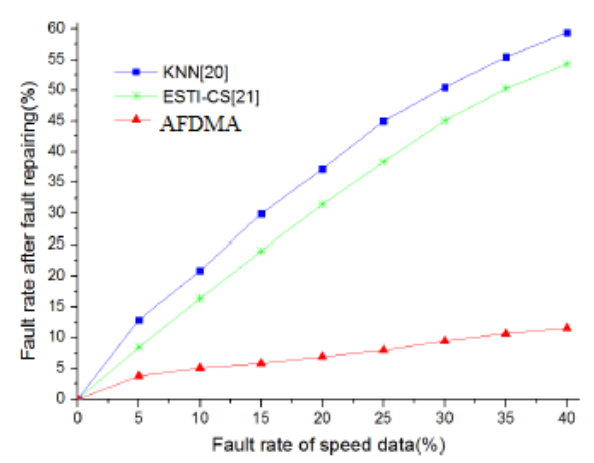

Figure 7. Fault identification based on vehicle speed at different locations

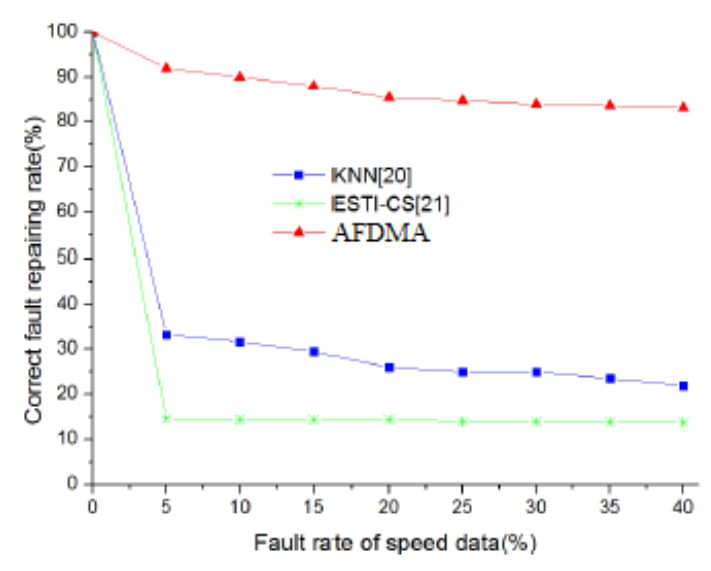

Figure 8. Performance of fault reduction and modification for vehicles

Based on above figures, we observe that proposed approach gives better and efficient results in terms of fault identification and modification in vehicle ad hoc networks. Compared with traditional approach K-NN, ESTI-CS proposed approach i.e. AFDMA gives better fault detection and modification performance in ad hoc networks. 


\section{CONCLUSION}

We describe and suggest the usage of Bayesian Network models for exploration of vehicle location data with an expanded Extended Bayesian (EBay) and Modified Approach (Expand) to identify and operationalize the location of current vehicle faults with real-time. We also discuss about Bayesian network mode with particle swarm optimization to reduce and identify the faults of vehicles in terms of tracking vehicle information and store their data. We use threshold based algorithm to provide efficient fault detect and modification procedure based on matrix formations. We evaluated the performance of proposed approach with respect to good fault identification and modification at low false rate. Further improvement of proposed approach to provide efficient solution from Sybil related attacks present in vehicular ad hoc networks, and also describe advanced index approaches to store encrypt data of vehicles.

\section{REFERENCES}

[1]. D. J. Hill, B. S. Minsker, and E. Amir, "RealTime Bayesian Anomaly Detection for Environmental Sensor Data", Water Resources Research, vol. 45, no. 4, pp. 450-455, 2007.

[2]. Wen Sun, Jiajia Liu, and Haibin Zhang, "When Smart Wearables Meet Intelligent Vehicles: Challenges and Future Directions", 15361284/17/\$25.00 @ 2017 IEEE.

[3]. Ford's New Wearables Lab Wants to Make Driving Your Car Safer with Sensors," 2016;: http://www.wareable.com/ cars/fords-newwearableslab-driving-safer-sensors-

2016,accessed Jan. 9, 2017.

[4]. H. Minh, A. Benslimane, and D. Deng, "Reliable Broadcasting Using Polling Scheme Based Receiver for Safety Applications in Vehicular Networks," IEEE Internet of Things J., vol.1, no. 4, 2014, pp. 289-99.
[5]. S. Woo, H. J. Jo, and D. H. Lee, "A Practical Wireless Attack on the Connected Car and Security Protocol for In-Vehicle CAN," IEEE Trans. Intelligent Transportation Systems, vol. 16, no. 5, 2015, pp. 993-1006

[6]. S. Kaplan et al., "Driver Behavior Analysis for Safe Driving: A Survey," IEEE Trans. Intelligent Transportation Systems, vol. 16, no. 6, 2015, pp. 3017-32.

[7]. K. Perera and D. Dias, "An Intelligent Driver Guidance Tool Using Location Based Services," Proc. 2011 IEEE Int'l. Conf. Spatial Data Mining and Geographical Knowledge Services, 2011.

[8]. A. Bujari, C. E. Palazzi, and D. Ronzani, "Multimedia Transmissions over Vehicular Networks," Proc. IEEE 27th Annual Int'l. Symp. Personal, Indoor, and Mobile Radio Commun., 2016.

[9]. Haibin Zhang, Qian Zhang, Jiajia Liu,"Fault Detection and Repairing for Intelligent Connected Vehicles Based on Dynamic Bayesian Network Model", 2018 IEEE. Personal use is permitted, but republication/redistribution requires IEEE permission.

[10]. A. Benslimane, "Localization in Vehicular Ad Hoc Networks", in Proceeding of Systems Communications, pp. 19-25, 2005.

[11]. F. Yang, S. Wang, J. Li, Z. Liu, and Q. Sun, “An overview of internet of vehicles", China Communications, vol. 11, no. 10, pp. 1-15, 2014.

[12]. W. Sun, J. Liu, and H. Zhang, "When smart wearable's meet intelligent vehicles: challenges and future directions", IEEE Wireless Communications Magazine, vol. 24, no. 3, pp. 58-65, 2017.

[13]. F. Zhou, and A. Benslimane, "Reliable safety message dissemination with minimum energy in VANETs", in Proceeding of Global 
Communications Conference, pp. 587-592, Cite this article as :

2014.

[14]. H. Zhang, J. Li, B. Wen, and J. Liu, "Connecting intelligent things in smart hospitals using NBIoT", IEEE Internet of Things Journal, 2018.DOI: 10.1109/JIOT.2018.2792423.

[15]. S. Yang, and Z. H. Liu, "Anomaly Detection for Internet Of Vehicles: A Trust Management Scheme With Affinity Propagation", in Proceeding of Mobile Information Systems, pp. 1-10, 2016.

[16]. Y. Lou, and Y. Dong, "Structure Learning Algorithm of DBN Based on Particle Swarm Optimization", in Proceeding of IEEE International Symposium on Distributed Computing and Applications for Business Engineering and Science, pp. 102-105, 2015.

[17]. G. Trabelsi, and P. Leray, "Benchmarking dynamic Bayesian network structure learning algorithms", in Proceeding of IEEE International Conference on Modeling, Simulation and Applied Optimization, pp. 1-6, 2013.

[18]. Kamakshaiah Kolli etc... A Traditional Analysis for Efficient Data Mining with Integrated Association Mining into Regression Techniques, 3rd International Conference on Communications and Cyber Physical Engineering, LNEE, volume 698,pp 1393-1404, ISBN: 978-981-15-7961-5.

[19]. Syed Umar et el., "Securities and threats of cloud computing and solutions" 2018 2nd International Conference on Inventive Systems and Control (ICISC), IEEE Explorer, Pages: 1162-1166.
Syed Umar, Tadele Debisa Deressa, Bodena Terfa, "A Modified Fault Detection Bayesian Learning Model For Inter Connected Vehicle Networks", International Journal of Scientific Research in Science, Engineering and Technology (IJSRSET), Online ISSN : 2394-4099, Print ISSN : 2395-1990, Volume 8 Issue 2, pp. 182-189, March-April 2021. Available at doi : https://doi.org/10.32628/IJSRSET218242 Journal URL : https://ijsrset.com/IJSRSET218242 